\title{
TTR
}

Traduction, terminologie, re?daction

\section{Au début était le traducteur}

\section{Antoine Berman}

Volume 14, numéro 2, 2e semestre 2001

Antoine Berman aujourd'hui

Antoine Berman for our time

URI : https://id.erudit.org/iderudit/000566ar

DOI : https://doi.org/10.7202/000566ar

Aller au sommaire du numéro

\section{Éditeur(s)}

Association canadienne de traductologie

ISSN

0835-8443 (imprimé)

1708-2188 (numérique)

Découvrir la revue

Citer cet article

Berman, A. (2001). Au début était le traducteur. TTR, 14(2), 15-18.

https://doi.org/10.7202/000566ar d'utilisation que vous pouvez consulter en ligne.

https://apropos.erudit.org/fr/usagers/politique-dutilisation/ 


\section{Au début était le traducteur ${ }^{1}$}

\section{Antoine Berman}

La réflexion sur la traduction est pratiquement née chez moi avec mon activité de traducteur. Elle en porte, plus ou moins directement, la marque. Mais elle est loin de n'être que l'explicitation conceptuelle, ou discursive, de cette activité. Je pense aujourd'hui que, fondamentalement, elle atteste un autre rapport à la traduction que le traduire lui-même; rapport qui, à sa manière, est aussi intense et intime que ce dernier. Tout se passe comme si, depuis mes débuts de traducteur, s'était manifesté le sentiment que, face à cette réalité qu'est la traduction, la simple « pratique » de la traduction ne me suffisait pas. Il me fallait aussi réfléchir, sans trêve, sur la traduction. Et cela non plus ne semble pas m'avoir suffi, puisqu'au fil des années, j'ai été attiré par l'enseignement, et de la pratique traductive, et de la réflexion traductologique.

Ce n'est pas tout : à l'époque même ou je commençais des séminaires de traduction au Collège international de philosophie (1984), j'ai été nommé «conseiller technique» au Commissariat général à la langue française, qui voulait créer un grand centre national de la traduction et de la terminologie. Bien que ce grand centre n'ait finalement pas vu le jour, j'ai été amené à prendre la direction de sa formule actuelle, le Centre Jacques-Amyot, dont l'objectif est de promouvoir en France les activités de traduction et de terminologie, c'est-à-dire de mener une « politique » (aussi limitée soit-elle) en faveur de ces activités. Ma réflexion sur la traduction s'accompagne donc de trois autres relations au traduire :

${ }^{1}$ Extraits d'un texte inédit, publiés grâce à l'aimable autorisation d'Isabelle Berman. 
- les traductions elles-mêmes (de diverses langues et de divers genres de textes);

- l'enseignement de la traduction et de la « traductologie »;

- l'activité «politique » (au sens large) en faveur de la traduction.

Puisque mon travail traductologique est inséparable de ces trois autres rapports à la traduction, il me semble utile, dans cette introduction, d'en dire quelques mots. Car bon nombre des textes que j'ai publiés - ouvrages, cours, articles, conférences, communications - ne se comprennent qu'en fonction de ces activités. Naturellement, et même si je ne traduis pas actuellement, il va sans dire que c'est l'expérience du traduire qui constitue le centre de gravité de mon rapport général à la traduction. Je ne suis traductologue que parce que je suis, primordialement, traducteur.

La pratique de la traduction n'exige nullement que l'on soit aussi un traductologue, si du moins l'on entend par là : théoricien de la traduction. Il est maints traducteurs qui se refusent de même à discourir sur leur pratique ou leur expérience. Et non des moindres. Mais il est également aisé de voir qu'aujourd'hui, de plus en plus, l'activité traduisante se soutient et s'accompagne d'une réflexion sur le traduire : que cette réflexion elle-même, se soutient et s'accompagne d'un désir de transmission, voire d'enseignement. Un certain narcissisme, un certain autisme du traduire - souvent liés à un anti-intellectualisme douteux - disparaissent peu à peu. Acte par excellence de transmission, la traduction se veut maintenant transmissible. J'appartiens, avec beaucoup d'autres, à cette génération de traducteurs soucieux de communiquer, d'ouvrir, de faire parler leur pratique. Cela n'implique pas a priori, de parti pris « théorique ».

Appartient à ce mouvement d'ouverture de la traduction le fait que, de plus en plus (et dans le monde entier), pratique traduisante et réflexion traductologique sont liées à l'espace de l'Université (au sens large). Cela ne signifie pas seulement qu'une proportion croissante de traducteurs littéraires soit des universitaires; ou plutôt ce fait indéniable renvoie à une réalité plus profonde. D'une part, l'espace universitaire est devenu (ce qu'il n'était pas de prime abord) un espace où traduction et pensée de la traduction peuvent s'épanouir; d'autre part, traduction et traductologie ne sont pas tout à fait, pour cet espace, des «matières » quelconques; l'assomption, par l'Université, de la traduction est peut- 
être de nature à lui permettre, par les chemins les plus divers, d'approcher davantage sa propre finalité, qui est la transmission. Inutile ici de s'étendre là-dessus : il me paraît évident que le lien de la traduction et de l'Université, tel qu'il se noue actuellement, est un phénomène historique important, où se jouera une bonne part du destin de ce qu'est la traduction et ce qu'on appelle, en Occident, l'Université. Et cela dépasse de beaucoup l'apparition, au sein des institutions empiriques, de «chaires» ou de «départements» de traductologie. $[\ldots]$

À Toulouse, dans une communication intitulée « La traduction de Moi le Suprême», je tentai de situer ma traduction dans le «mouvement» évoqué plus haut, de préciser le concept du traduire et de 1'échange culturel qui avait préside à mon travail, de détailler les principes choisis pour traduire et, enfin d'illustrer l'application de ces principes par quelques exemples que je jugeais à la fois significatifs, accomplis et distrayants. Lors de cette communication, qui fut fort bien accueillie, je pris pour la première fois la mesure de ce que pouvaient être une parole et une pensée de la traduction. À ma fascination pour le phénomène du traduire pouvait correspondre, chez l'auditeur, chez le lecteur, une égale fascination, si du moins l'on parvenait à rendre la traduction passionnante. Et il est devenu clair pour moi au fil des années que parler et écrire sur la traduction, c'est cela : la rendre, pour les autres, passionnante. Mais cela n'est possible que si le «discours traductologique » parvient à faire scintiller (comme aurait dit Michel Foucault) la dimension de la traduction dans toute sa multiplicité, sa profondeur et son obscurité. Parler de traduction, c'est parler des œuvres, de la vie, du destin et de la nature des œuvres; de la manière dont elles éclairent nos vies; c'est parler de la communication, de la transmission, de la tradition; c'est parler du rapport du Propre et de l'Étranger; c'est parler de la langue maternelle, natale, et des autres langues; c'est parler de l'être-en-langues de l'homme; c'est parler de l'écriture et de l'oralité; c'est parler du mensonge et de la vérité, de la trahison et de la fidélité; c'est parler du mimétique, du double, du leurre, de la secondarité; c'est parler de la vie du sens et de la vie de la lettre; c'est... être pris dans un enivrant tourbillon réflexif où le mot « traduction » lui-même ne cesse de se métaphoriser.

Tel fut l'espace qui s'ouvrit à moi après la traduction de Moi le Suprême et après la transmission, à Toulouse, de l'expérience de cette traduction. Il m'était clair, toutefois, qu'une réflexion sur la traduction, tout en se fondant sur l'expérience du traduire, ne pouvait en aucun cas 
s'y limiter et en être la simple explicitation. Il y avait place pour une pensée sui generis qui produirait d'autant mieux l'« essence» de la traduction qu'elle ne s'absorberait pas dans la description de ses «problèmes" empiriques. Ou, pour le dire autrement - il fallait concevoir de manière non restrictive l'expérience qu'était la traduction : en faire une expérience humaine fondamentale, digne, comme toutes les expériences humaines fondamentales, d'être pensée.

Il pouvait certes exister (et il existe) une traductologie scientifique, objective, positive, débouchant sur des « méthodologies »; il pouvait exister aussi, sur un mode discursif, mais non proprement théorique, une traductologie " essayistique » et même, à son extrémité, «spéculative». La première avait les limites de toute visée scientifique; la seconde, la dangereuse absence de limites de la réflexion. Toutes deux, dans leur différence, sont probablement les deux figures antagonistes du savoir moderne de la traduction. Par trajectoire, penchant et tempérament, j'ai opté pour une traductologie « essayistique ». 